\title{
Present Scenarios of Otomycosis in Rajshahi City of Bangladesh
}

\author{
Karishma Kabir Nipa ${ }^{1}$, AHM Mostafa Kamal ${ }^{2}$, Muhammad Jakir Hossain ${ }^{3}$, Ahmed Imtiaj ${ }^{4, ~ * ~}$ \\ ${ }^{1}$ Institute of Biological Science, University of Rajshahi, Rajshahi, Bangladesh \\ ${ }^{2}$ Intensive Care Unit, Rajshahi Medical College Hospital, Rajshahi, Bangladesh \\ ${ }^{3}$ Department of Agricultural Genetic Engineering, Nigde Omer Halisdemir University, Nigde, Turkey \\ ${ }^{4}$ Department of Botany, University of Rajshahi, Rajshahi, Bangladesh
}

Email address:

aimtiajbot@ru.ac.bd (A. Imtiaj)

${ }^{*}$ Corresponding author

To cite this article:

Karishma Kabir Nipa, AHM Mostafa Kamal, Muhammad Jakir Hossain, Ahmed Imtiaj. Present Scenarios of Otomycosis in Rajshahi City of Bangladesh. American Journal of Biomedical and Life Sciences. Vol. 8, No. 5, 2020, pp. 137-142. doi: 10.11648/j.ajbls.20200805.13

Received: July 23, 2020; Accepted: August 18, 2020; Published: September 10, 2020

\begin{abstract}
In current study, an attempt was taken to investigate into the scenarios of otomycosis disease in Rajshahi metropolitan city of Bangladesh. There were 237 otomycosis patients found among 3875 ear patients during the studied period. Habit, habitat, life style, personal hygiene and food or nutritional status were found to be crucial factors which were largely responsible for the onset of otomycosis. Among 237 otomycosis patients, 125 and 112 sufferers were from rural and urban regions, respectively. In rural, male was more sufferer than female whereas, in urban, result was opposite to rural. Among 3 consecutive seasonal periods, the incidence of otomycosis patients was the highest in humid/rainy season $(50.6 \%)$, intermediate level in winter season $(35.4 \%)$ and the lowest level in dry seasons $(14.0 \%)$. No significant variation was found between male $(50.2 \%)$ and female $(49.8 \%)$ patients for the onset of otomycosis. Age group was also potential for the onset of studied disease. The occurrences of over age (61 to above), baby (up to 10) and juvenile (11 to 20 ) group were $30.4 \%, 27.8 \%$ and $27.4 \%$, respectively which were high. Whereas, the incidence was found to be low in early youth $(5.1 \%)$ and late youth (9.3\%) groups.
\end{abstract}

Keywords: Associated Complications, Ear Canal, Factors, Fungal Infection, Otomycosis

\section{Introduction}

Human ear is the most important sensor to detect sound signal at frequencies from about $20 \mathrm{~Hz}$ to $20,000 \mathrm{~Hz}$, though we hear sounds best from $1,000 \mathrm{~Hz}$ to $5,000 \mathrm{~Hz}$, where sound of human speech is optimized.

This central natural device is often being infected by enormous microorganism e. g. Aspergillus spp., Candida spp. and also some bacterial species. It is very difficult to diagnose and treat due to diversified and nonspecific symptoms as well as common recurrence including hearing loss may reduce the range of frequencies a person can hear. Otomycosis is one of the major ear infections which known as superficial mycotic disease of the outer ear canal. External ear infection is a common inflammation of the external ear canal and auricle that occurs due to various local infectious factors or a manifestation of a systemic disease along with discomfort, tinnitus, hearing impairment and discharge [15]. About $10 \%$ people suffer from infection of the external ear canal in their lives, $90 \%$ of which is unilateral $[17,3]$. It is also called otitis external often involving the pinna and the external auditory meatus, however in the presence of a perforated tympanic membrane; it can also involve the middle ear [16]. The mastoid cavity can also be involved following open cavity mastoidectomy. The main symptoms include pruritus, otalgia, aural fullness, hearing impairment, otorrhea and tinnitus. The prevalence of otomycosis is related to the socioeconomic condition and geographic area with higher rates in tropical and subtropical climates [9]. In recent years, these opportunistic fungal infections have gained 
greater importance in human medicine and probably due to the increasing number of immune compromised patients are responsible for this higher incidence [29]. Immunocompetent hosts [8]. also can be invaded by this fungal infection manifested by complications such as hearing loss and invasive temporal bone infection [23, 24]. Patients with diabetes, lymphoma, or AIDS and patients undergoing or receiving chemotherapy or radiation therapy are at increased risk for potential complications for otomycosis [29] and in severe cases it can be manifested by hemorrhagic granulations can cause thrombosis of adjacent blood vessels leading to avascular necrosis and perforation of TM [23].

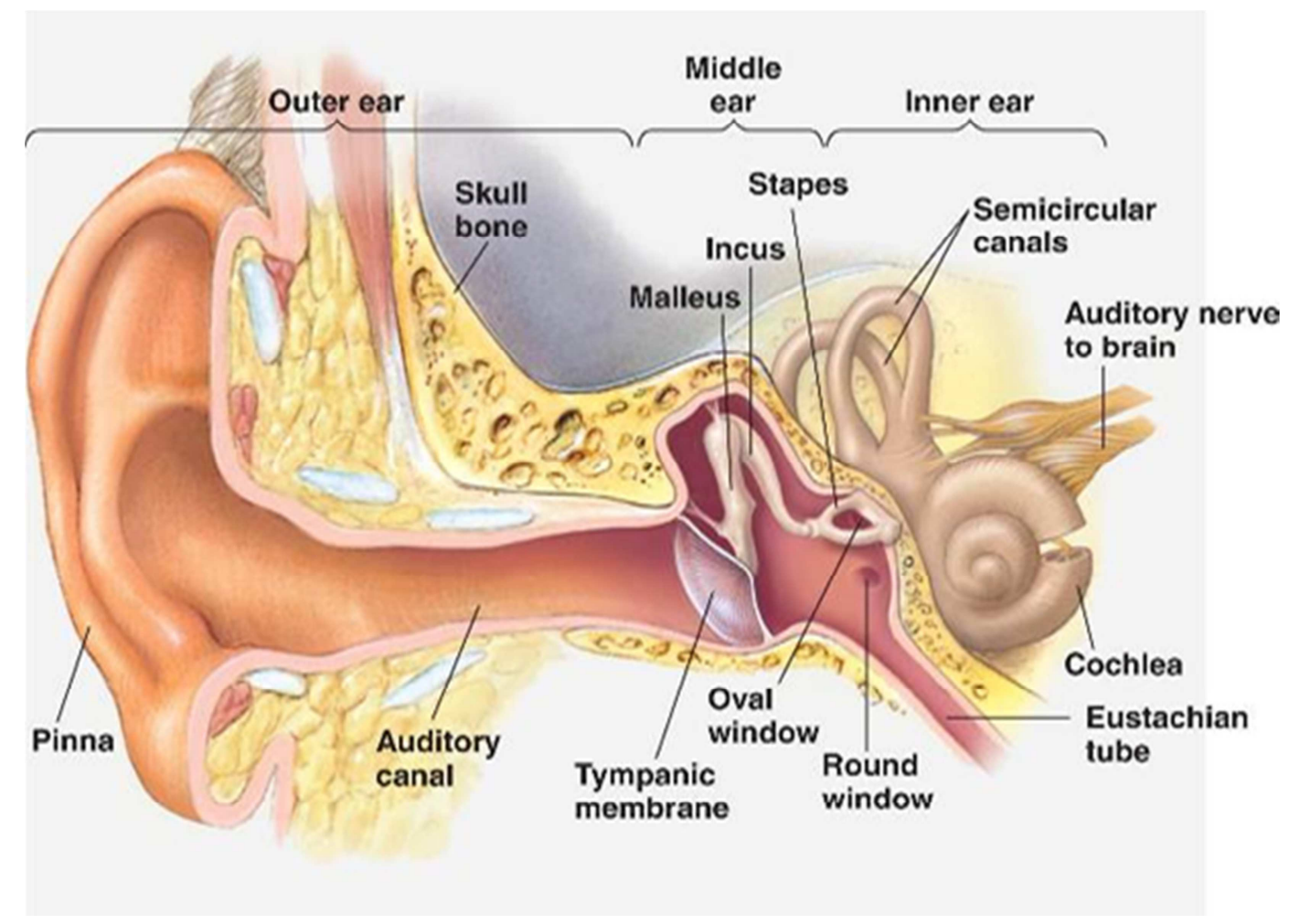

Figure 1. The anatomy of the auditory system of human ear.

Previous study revealed that in most of cases this fungal infection was caused by either Aspergillus or Candida species [21, 29] however, other species such as dermatophytes and Mucor were also isolated [13, 14]. Due to the dispersal of spores from those airborne fungi, the incidence of infection was raised in high level and approximately over 200 species can affect human [25]. Influencing factors include habitual instrumentation, dermatitis, immunocompromising conditions or alterations in immunity, use of steroids, dermatological diseases, loss of cerumen, use of broad-spectrum antibiotics and hearing aids. Beside the above-mentioned factors, changes in epithelial covering increase in $\mathrm{pH}$ (bathing), systemic factors (debilitating disease and neoplasia), environmental factor, chronic secretory otitis media, history of bacterial infections etc. can instigate the entire disease cycle [19]. Literature reveals that most of the studies about the etiology of otomycosis have been carried out in tropical and subtropical areas. Etiology of fungal pathogens in dry dusty regions is not similar to hot humid areas and this needs to be considered in future susceptibility tests and treatment of patients with otomycosis. That is why; this study was carried out to evaluate the features of otomycosis in Rajshahi Metropolitan City (RMC) which represents both dry dusty and hot humid region of Bangladesh.

\section{Materials and Methods}

\subsection{Survey}

The survey was done attending the patients in the out-door and ward of Rajshahi Medical College Hospital (RMCH), Non-Government Medical College Hospitals (NGMCH), Non-Government Diagnostic Centers (NGDC) and Selfobservation (SO) in/around Rajshahi Metropolitan City (RMC) between January 2018 and December 2018. To collect data patients, doctors, doctor's attendants, nurses, chemists of drug centers and representatives of medicine companies were visited.

\subsection{Data Collection}

All patients having ear diseases those who visited the above-mentioned hospitals and diagnostic centers during the time frame were taken in consideration for the study. Among them, patients of only otomycosis were separated for further investigation to collect data regarding otomycosis. An oral questionnaire without personal identity for privacy reason was used to collect the necessary data. 


\subsection{Samples Collection and Culture Condition}

Clinical manifestations of patients were including otalgia, otorrhea, tinnitus, pruritis and hearing impairment. Mycological study was carried out on debris, scrapping or exudates samples from auditory canal (s). The sample collection was obtained from patients of otomycosis with the help of a sterile cotton swab from the external auditory canal or a sterile scalpel blade. All samples were initially preserved below $10^{\circ} \mathrm{C}$ to avoid overgrowth of saprophytic mycoflora. For each case, 4 replicates of a sample were preserved in a refrigerator. The samples were sown in the laboratory and inoculated on 3 different media: Sabouraud chloramphenicol agar, blood agar and Malt yeast agar. Each sample swab was rolled on all 3 media and inoculated at $30^{\circ} \mathrm{C}$ for 2 weeks. All samples were evaluated both direct examination and culture method. Direct examination of samples was done using glass sliders treated with $10 \% \mathrm{KOH}$ as optical brightener and lactophenol. Using the criteria of Loddler [12] and Hoog and Guarro [10] mycological identification was done.

\section{Results and Discussion}

Five major hospitals from the study area were selected to investigate into the scenarios of different ear diseases including otomycosis in Rajshahi metropolitan city. A total of 3875 sufferers from varieties of ear diseases were visited any of the five hospitals between January 2018 and December 2018. Of 3875,237 patients were found to be otomycosis disease which was average $5.6 \%$. Rests of 3638 patients were suffered from different ear diseases other than nonotomycosis such as aural pruritis, otalgia, ear discharge, headache, hearing impairment and aural blockage. The highest and lowest incidences of non-otomycosis were recorded for otalgia (38\%) and aural blockage (4\%), respectively. The incidence of aural pruritis was also found to be high (31\%). Among the 5 hospitals, out-door of RMCH was covered maximum number of non-otomycosis and otomycosis patients with the highest percentage $(29 \%$ and $7 \%$, respectively). Whereas $\mathrm{AH}$ was covered minimum number of non-otomycosis and otomycosis patients with the lowest percentage (11\% and 5\%, respectively). Aneja [1] reported that $78 \%$ of the patients were positive for otomycosis whereas Kaur [11] reported that $74.7 \%$ patients were suffered from otomycosis. Ozcan [18], Chin and Jegathesanin [5] and Pontes [22] revealed that 65\%, 74.6\% and $19.4 \%$ patients were found to be suffered from otomycosis disease in their study.

Table 1. Scenarios of ear diseases including otomycosis in Rajshahi city.

\begin{tabular}{|c|c|c|c|c|c|c|c|c|}
\hline \multirow[b]{2}{*}{ Hospital } & \multicolumn{6}{|c|}{ Number of patient's in different clinical conditions } & \multirow{2}{*}{$\begin{array}{l}\text { Total Patients } \\
\text { Attended }\end{array}$} & \multirow{2}{*}{$\begin{array}{l}\text { Patient's no. of } \\
\text { Otomycosis }\end{array}$} \\
\hline & $\begin{array}{l}\text { Aural } \\
\text { Pruritis }\end{array}$ & Otalgia & $\begin{array}{l}\text { Ear } \\
\text { Discharge }\end{array}$ & Headache & $\begin{array}{l}\text { Hearing } \\
\text { Impairment }\end{array}$ & $\begin{array}{l}\text { Aural } \\
\text { Blockage }\end{array}$ & & \\
\hline ORMCH & 348 & 426 & 135 & 68 & 101 & 45 & $1123(29 \%)$ & $83(7 \%)$ \\
\hline WRMCH & 190 & 233 & 73 & 37 & 55 & 24 & $612(16 \%)$ & $32(5 \%)$ \\
\hline $\mathrm{NGMCH}$ & 272 & 334 & 105 & 53 & 79 & 35 & $878(23 \%)$ & $57(6 \%)$ \\
\hline NGDC & 258 & 317 & 100 & 50 & 75 & 33 & $833(21 \%)$ & $43(5 \%)$ \\
\hline SO & 133 & 163 & 51 & 26 & 39 & 17 & $429(11 \%)$ & $22(5 \%)$ \\
\hline
\end{tabular}

ORMCH: Out-door of Rajshahi Medical College Hospital, WRMCH: Ward of Rajshahi Medical College Hospital, NGMCH: Non-Government Medical College Hospitals, NGDC: Non-Government Diagnostic Centers and SO: Self-Observation.

From Table 1 there could be a logical question why the highest number of patients visited out-doors of RMCH? The possible answer of the question could be that $\mathrm{RMCH}$ is a public hospital and mostly poor people prefer to this hospital for their treatment paying low cost. Rests of the hospitals are private and usually financially solvent people go there to ensure medication paying high cost. So, it can be speculated that prevalence of ear infection as well as otomycosis may related to the life style and hygiene condition.

Habitual practices and life style of the target groups were the most prominent trigger factor of otomycosis in the study area. As Rajshahi is a hot and humid area with densely distributed population residing with remarkable middle and low class of citizen including slum dwellers, day laborers and beggars surrounded by several districts and sub-districts from which people of different economic classes and life styles attend to the studied hospitals for their checkup and medication. Above mentioned factors are largely responsible for the onset of otomycosis. Among 237 otomycosis patients, 125 and 112 patients were from rural and urban region of
Rajshahi, respectively (Table 2).

Table 2. Habitat and gender distribution of otomycosis patients.

\begin{tabular}{|c|c|c|c|c|c|}
\hline \multirow{3}{*}{ Hospital/Clinic } & \multicolumn{4}{|l|}{ Habitat } & \multirow{3}{*}{ Total } \\
\hline & \multicolumn{2}{|l|}{ Rural } & \multicolumn{2}{|l|}{ Urban } & \\
\hline & Female & Male & Female & Male & \\
\hline$\overline{\mathrm{ORMCH}}$ & 21 & 20 & 25 & 17 & 83 \\
\hline WRMCH & 08 & 11 & 07 & 06 & 32 \\
\hline NGMCH & 12 & 17 & 14 & 14 & 57 \\
\hline NGDC & 10 & 15 & 11 & 07 & 43 \\
\hline SO & 04 & 07 & 06 & 05 & 22 \\
\hline Total & $\begin{array}{l}55 \\
125\end{array}$ & 70 & $\begin{array}{l}63 \\
112\end{array}$ & 49 & 237 \\
\hline
\end{tabular}

ORMCH: Out-door of Rajshahi Medical College Hospital, WRMCH: Ward of Rajshahi Medical College Hospital, NGMCH: Non-Government Medical College Hospitals, NGDC: Non-Government Diagnostic Centers and SO: Self-Observation.

Current study depicts that the incidence of otomycosis was slightly frequent among the rural participants compared with the urban people. Personal hygiene practices and nutritional status in daily food habit can be the probable cause of the 
findings. Present studies also denote that, females were more sufferers from the otomycosis than the males. The findings of our present study do not correlate the findings of Than et al. [28] on which it was showed $52 \%$ in males and $48 \%$ in females whereas in our study, the incidence of otomycosis was more among the females (55 out 125 in rural area and 63 out of 112 in urban area) than the males (70 out of 125 in rural area and 49 out of 112 in urban area) patients. Our study revealed that in normal and urban condition females are more victims but in rural areas males are more sufferers from otomycosis. Pontes et al. [22], Aneja et al. [1], Ozcan et al. [18] and Ghiacei et al. [8] mentioned that female was more susceptible to the otomycosis. Otomycoses was frequent in tropical and subtropical climates because of heat and humidity [20, 27, 30].

Table 3. Season and gender distribution of otomycosis patients.

\begin{tabular}{|c|c|c|c|c|}
\hline \multirow{2}{*}{ Hospital } & \multirow{2}{*}{ Season } & \multicolumn{2}{|l|}{ No. of patient } & \multirow{2}{*}{ Total } \\
\hline & & Male & Female & \\
\hline \multirow{3}{*}{$\mathrm{ORMCH}$} & Winter Season (Nov - Mar) & 14 & 18 & $32(13.5 \%)$ \\
\hline & Dry Season (Apr - Jun) & 5 & 7 & $12(5.1 \%)$ \\
\hline & Humid/Rainy Season (Jul - Octo) & 18 & 21 & $39(16.5 \%)$ \\
\hline \multirow{3}{*}{ WRMCH } & Winter Season (Nov - Mar) & 5 & 6 & $11(4.6 \%)$ \\
\hline & Dry Season (Apr - Jun) & 3 & 1 & $4(1.7 \%)$ \\
\hline & Humid/Rainy Season (Jul - Octo) & 9 & 8 & $17(7.2 \%)$ \\
\hline \multirow{3}{*}{ NGMCH } & Winter Season (Nov - Mar) & 11 & 10 & $21(8.9 \%)$ \\
\hline & Dry Season (Apr - Jun) & 4 & 4 & $8(3.4 \%)$ \\
\hline & Humid/Rainy Season (Jul - Octo) & 16 & 12 & $28(11.8 \%)$ \\
\hline \multirow{3}{*}{ NGDC } & Winter Season (Nov - Mar) & 8 & 6 & $14(5.9 \%)$ \\
\hline & Dry Season (Apr - Jun) & 2 & 4 & $6(2.5 \%)$ \\
\hline & Humid/Rainy Season (Jul - Octo) & 12 & 11 & $23(9.7 \%)$ \\
\hline \multirow{4}{*}{ SO } & Winter Season (Nov - Mar) & 3 & 3 & $6(2.5 \%)$ \\
\hline & Dry Season (Apr - Jun) & 2 & 1 & $3(1.3 \%)$ \\
\hline & Humid/Rainy Season (Jul - Octo) & 7 & 6 & $13(5.5 \%)$ \\
\hline & Total & $119(50.2 \%)$ & $118(49.8 \%)$ & $237(100 \%)$ \\
\hline
\end{tabular}

ORMCH: Out-door of Rajshahi Medical College Hospital, WRMCH: Ward of Rajshahi Medical College Hospital, NGMCH: Non-Government Medical College Hospitals, NGDC: Non-Government Diagnostic Centers and SO: Self-Observation.

To measure the seasonal impact on otomycosis, 5 major hospitals in our study area and 3 consecutive seasonal periods (Winter: November to March, Dry: April to June and Humid/Rainy: July to October) were taken in consideration. Among the seasonal periods, the incidence of otomycosis patients was the highest in humid/rainy season (50.6\%), intermediate level in winter season $(35.4 \%)$ and the lowest level in dry seasons $(14.0 \%)$. No significant variation was found between male $(50.2 \%)$ and female $(49.8 \%)$ patients for the onset of otomycosis (Table 3). Aneja et al. [1] and Ozcan et al.[18] mentioned that hot and humid weather condition of summer was more suitable for the growth, reproduction and epidemic formation of fungal infections. Highest prevalence of otomycosis in summer has been reported by Paulose et al. [21], Ozcan et al. [18], Ghiacei et al. [8] and Pontes et al. [22].

Table 4. Occurrence of otomycosis in different age groups of patients.

\begin{tabular}{|c|c|c|c|c|c|}
\hline \multirow{2}{*}{$\begin{array}{l}\text { Hospital/Diagnostic } \\
\text { Center }\end{array}$} & \multicolumn{5}{|c|}{ Age interval (years) } \\
\hline & 0-10 (Baby) & 11-20 (Juvenile) & 21-40 (Early youth) & 41-60 (Late youth) & 61-above (Over age) \\
\hline ORMCH & 22 & 23 & 6 & 8 & 24 \\
\hline WRMCH & 9 & 9 & 0 & 3 & 11 \\
\hline NGMCH & 17 & 15 & 3 & 5 & 17 \\
\hline NGDC & 11 & 13 & 2 & 4 & 13 \\
\hline SO & 7 & 5 & 1 & 2 & 7 \\
\hline Total & $66(27.8 \%)$ & $65(27.4 \%)$ & $12(5.1 \%)$ & $22(9.3 \%)$ & $72(30.4 \%)$ \\
\hline
\end{tabular}

ORMCH: Out-door of Rajshahi Medical College Hospital, WRMCH: Ward of Rajshahi Medical College Hospital, NGMCH: Non-Government Medical College Hospitals, NGDC: Non-Government Diagnostic Centers and SO: Self-Observation.

Age intervals among the patients showed potential difference in disease prevalence and present study revealed that people from any age groups were prone to the mycotic infection and patients from one year old to 80 years can be suffered from otomycosis. As shown in Table 4, out of 237 patients, $66(27.8 \%)$ and $72(30.4 \%)$ cases were recorded in the age group 0 to 10 (babies) and 61 to above (over age), respectively. Whereas, the incidence was found to be low above 21 years and below 60 years. In our study the incidence was very low (12 which was $5.1 \%$ ) in the agegroup 21-40 years which is called early youth. According to Aneja et al. [1], Fasunla et al. [7], Singh et al. [26] and Pontes et al. [22] otomycosis was observed more often between the age group 21-40 years old and had a higher incident in females than males. In case of age group our findings do not correlate but gender group matches between the studies. As we know immune systems of baby and over aged people are usually immature and defected and these 
conditions are susceptible to pathogens. That is why, early and late age was the main cause of high prevalence of otomycosis.

Table 5. Findings from KOH preparation and culture.

\begin{tabular}{llll}
\hline Positivity & Culture Positive & Culture Negative & Total \\
\hline $\mathrm{KOH}^{+\mathrm{ve}}$ & $158(66.7 \%)$ & $38(16.0 \%)$ & $196(82.7 \%)$ \\
$\mathrm{KOH}^{-\mathrm{ve}}$ & $7(2.9 \%)$ & $34(14.4 \%)$ & $41(17.3 \%)$ \\
Total & $165(69.6 \%)$ & $72(30.4 \%)$ & 237 \\
\hline
\end{tabular}

In current study, $165(69.6 \%)$ and $72(30.4 \%)$ patients were found mycological positive and negative respectively during the test of culture condition. Of 237, 158 samples were culture positive and 38 samples were culture negative against $\mathrm{KOH}^{+\mathrm{ve}}$ that was high compared with the data found against $\mathrm{KOH}^{-\mathrm{ve}}$ (Table 5).

Table 6. Percentages of fungal flora on culture positive samples.

\begin{tabular}{lllll}
\hline \multirow{2}{*}{ Fungal isolates } & No. of & \multirow{2}{*}{$\begin{array}{l}\text { \%of } \\
\text { isolates }\end{array}$} & isolates & Fender \\
\cline { 4 - 5 } & 80 & $48.2 \%$ & 42 & 38 \\
\hline Aspergillus flavus & 60 & $36.4 \%$ & 35 & 25 \\
Aspergillus niger & 13 & $7.9 \%$ & 8 & 5 \\
Candida albicans & 7 & $4.5 \%$ & 4 & 3 \\
Aspergillus fumigatus & 3 & $2.0 \%$ & 1 & 2 \\
Aspergillus nidulans & 2 & $1.0 \%$ & 2 & 0 \\
Candida parapsilosis & 2 & $100 \%$ & 88 & 77 \\
Total & 165 & & & \\
\hline
\end{tabular}

The present investigation revealed the presence of several fungi as causal agents of otomycosis. In terms of Aspergillus positive isolates, Aspergillus flavus was the most common and its account for otomycosis was $80(48.2 \%)$ followed by A. niger (36.4\%), A. fumigates (4.5\%) and A. nidulans (2\%). As responsible pathogen, Candida species was also noted which constituted $4.5 \%$ and $1 \%$ of fungal isolates, respectively (Table 6). Araiza et al. [2] also reported A. flavus was to be the most common pathogen in Mexico. Kaur et al. [11] reported that $A$. fumigates was the most common cause of otomycosis. Darko et al. [6] and Pontes et al. [22] reported Candida genus as the predominant pathogen in otomycosis. There can be a question made whether any pathogen is identified as primary or secondary agent especially when multiple organisms are isolated from a culture. To saturate this question, we think more progressive research is required.

\section{References}

[1] Aneja, KR, C Sharma and R Joshi, 2010. Fungal infection of the ear: a common problem in the north eastern part of Haryana. Int'l J. Pediatric Otorhinolaryngology, 74: 604-7.

[2] Araiza, J, P Canseco and A Bonifaz, 2006. Otomycosis: clinical and mycological study of 97 cases. Rev Laryngol Otol Rhinol (Bord), 127: 251-4.

[3] Beers, SL and TJ Abramo, 2004. Otitis externa review. Pediatr Emerg Care, 20: 250-6.

[4] Carney, AS, 2008. Otitis externa and otomycosis. In: Gleeson
MJj Jones NS, Clarke R, et al. (eds). Scott-Brown's Otolaryngology, Head and Neck Surgery, vol 3, 7th edn. London: Hodder Arnold Publishers; 2008: 3351-7.

[5] Chin, CS and M Jegathesan, 1982. Fungal isolates in otomycosis. Malays J. Pathol., 5: 45-7.

[6] Drako, E, A Jenca, M Orencak, S Viragova and E Pilipcinec, 2004. Otomycosis of Candidal origin in eastern Slovakia. Folia Microbiol (Praha), 49: 601-4.

[7] Fasunla, J, T Ibekwe and P Onakoya, 2007. Otomycosis in Western Nigeria. Mycoses, 51: 67-70.

[8] Ghiacei, S, 2001. Survey of otomycosis in north-western area of Iran. Med J. Mashhad Uni Med Sci., 43: 85-7.

[9] Ho, T, JT Vrabec, D Yoo and NJ Coker, 2006. Otomycosis: clinical features and treatment implications. Otolaryngol Head Neck Surg., 135: 787-91.

[10] Hoog, GS and I Guarro, 1995. Atlas of clinical fungi. The Netherlands: Central bureau of Schimmel cultures.

[11] Kaur, R, N Mittal, M Kakkar, AK Aggarwal and MD Mathur, 2000. Otomycosis: a clinicomycologic study. Ear Nose Throat J., 79: 606-9.

[12] Loddler, J, 1971. The yeasts: a taxonomic study. 2nd edition. Amsterdam: North-Holland Publish Company.

[13] Martin, TJ, JE Kerschner and VA Flanary, 2005. Fung al causes of otitis externa and tympanostomy tube otorrhea. Int'l J. pediatric Otorhino laryngology., 96: 1503-1508.

[14] Mishra, GS, N Mehta and M Pal, 2005. Chronic bilateral otomycosis caused by Aspergillus niger. Mycosis, 47: 82-84.

[15] Mofatteh, MR, ZN Yazdi, M Yousefi and MH Namaei, 2017. Comparison of the recovery rate of otomycosis using betadine and clotrima-zole topical treatment. Braz J Otorhinolaryngol. http://dx.doi.org/10.1016/j.bjorl.2017.04.004.

[16] Munguia, R and SJ Daniel, 2008. Ototopical antifungals and otomycosis: a review. Int J Pediatr Otorhinolaryngol., 72: 453-9.

[17] Ong, Y and G Chee, 2005. Infections of the external ear. Ann Acad Med Singapore, 34: 330-4.

[18] Ozcan, MK, M Ozcan, A Karaarslan and F Karaarslan, 2003. Otomycosis in Turkey: predisposing factors, etiologyand therapy. J. Laryngol Otol., 117: 39-42.

[19] Pahwa, VK, PC Chamiyal and PN Suri, 1983. Mycological study in otomycosis. Indian J. Med Res., 77: 334-8.

[20] Pardhan, B, NR Tuladhar and RM Amatya, 2003. Prevalence of otomycosis in outpatient department ofotolaryngology in Tribhuvan University Teaching Hospital, Kathmandu, Nepal. Ann OtolRhinolLaryngol., 112: 384-7.

[21] Paulose, KO, S Al-Khalifa, P Shenoy and RK Sharma, 1989. Mycotic infection of the ear (otomycosis): a prospective study. J. Laryngol Otol., 103: 30-5.

[22] Pontes, ZB, AD Silva, E Lima, M Guerra, N Oliviera, M Carvalho and FS Guerra, 2009. Otomycosis: a retrospective study. Braz J. Otorhinolaryngol., 75: 367-70.

[23] Rama Kumar, K, 1984. Silent perforation of tympanic membrane and otomycosis. Indian Journal of Otolaryngology and Head and Neck Surgery, 36 (4): 161-2. 
[24] Rutt, AL and RT Sataloff, 2008. Aspergillus otomycosis in an immunocompromised patient. Ent. J., 87 (2): 622-3.

[25] Shadzi, SH, 1988. Medical mycology, 3rd ed, Tehran, Gostaresh Farhang Press, p 337.

[26] Singh, S, H Singh and A Kaur, 2017. Otomycosis: A Clinical and Mycological Study. Indian Journal of Basic and Applied Medical Research, 6 (2): 271-5.

[27] Stern, JC and FE Lucente, 1988. Otomycosis. Ear Nose Throat J., 67: 804-10.
[28] Than, KM, KS Naing and M Min, 1980. Otomycosis in Burma and its treatment. Am. J. Trop. Med. Hyg., 29 (4): 620623.

[29] Viswanatha, B et al. 2012. Otomycosis in immunocompetent and immunocompromised patients; comparative study and literature review, Ent. J., 91 (3): 114-21.

[30] Yehia, MM, HM Al-Habib and NM Shehab, 1990. Otomycosis: a common problem in North Iraq. J. Laryngol. Otol., 104: 387-9. 